Notre Dame Journal of Formal Logic

Volume 39, Number 1, Winter 1998

\title{
A Variant of Thomason's First-Order Logic CF Based on Situations
}

\author{
XUEGANG WANG and PETER MOTT
}

\begin{abstract}
In this paper, we define a first-order logic $\mathbf{C} \mathbf{F}^{\prime}$ with strong negation and bounded static quantifiers, which is a variant of Thomason's logic $\mathbf{C F}$. For the logic $\mathbf{C} \mathbf{F}^{\prime}$, the usual Kripke formal semantics is defined based on situations, and a sound and complete axiomatic system is established based on the axiomatic systems of constructive logics with strong negation and Thomason's completeness proof techniques. With the use of bounded quantifiers, $\mathbf{C F}^{\prime}$ allows the domain of quantification to be empty and allows for nondenoting constants. $\mathbf{C F}^{\prime}$ is intended as a fragment of a logic for situation theory. Thus the connection between $\mathbf{C F}^{\prime}$ and infon logic is discussed.
\end{abstract}

1 Introduction Thomason [25] constructed a first-order logic CF. In his logic, a constructive negation is used instead of a classical or intuitionistic one. Constructive negation, also called strong negation, was introduced by Nelson 21 following Kleene's notion of recursive realizability, emphasizing that false number-theoretic statements, as well as true ones, are obtained simultaneously by constructive means. Independently, Markov [19] also introduced strong negation from the point of view of constructive logic. Such negation was later incorporated into various logical systems, such as Nelson's propositional systems $\mathbf{N}$ and $\mathbf{N}_{\mathbf{1}}$, that is, the propositional parts of Nelson's system $\mathbf{N}_{\mathbf{1}}$ of constructible falsity (see [21] and Routley [24]), ${ }^{1}$ and their first-order extensions (see Almukdad and Nelson [4]), ${ }^{2}$ intuitionistic logic with strong negation $\overline{\mathbf{H}}$ by Gurevich [17], constructive predicate logic with strong negation $\mathbf{S}$ by Akama [2], and first-order logic $\mathbf{C F}$ in 25]. See also constructive propositional calculus with strong negation by Vorob'ev [30] and the semantics of the calculus in terms of $\mathcal{N}$-lattices in Rasiowa [22]. Furthermore, Wansing [32] has systematically investigated the whole family of substructural subsystems of Nelson's systems from the point of view of the fine-structure of information processing. ${ }^{3}$

The resulting logics, to which we shall refer loosely as constructive logics with strong negation, demonstrate some satisfying features compared with intuitionistic 
logics. First of all, since negative information is treated as of equal importance with positive information, such logics are more symmetrical than intuitionistic logics and satisfy very natural duality laws. In particular, strong negation avoids nonconstructive features possessed by intuitionistic negation (see [17] and 32]). Secondly, constructive logics with strong negation can be provided with a more satisfying interpretation than the well-known Brouwer-Heyting-Kolmogorov (BHK) interpretation for intuitionistic logics (see [32] and Lopez-Escobar [18]). Moreover, they admit a sentence to be undetermined and thus can accommodate the partiality of information (see [25] and [32]).

Another desirable characteristic of constructive logics with strong negation is the heredity or persistence of information, ${ }^{4}$ to the effect that what is true at a state of information is still true at all later states. This is usually bought at the cost of a very strong dynamic satisfaction condition on universal quantifiers. A sentence $\forall x \varphi(x)$ is true at a state of information $s$ only when $\varphi(\underline{a})^{5}$ is true at all states of information $t \geq s$ for all individuals $a$ in the domain of $t$ (where $\geq$ orders states by increasing information).

Classically we should evaluate the quantifier in a static fashion where only the state $s$ and the individuals in the domain of $s$ are relevant. Which form is more natural from the situation-theoretic viewpoint? Consider the situation $s$ of a room full of people. The sentence 'All men here are hungry' will be true at $s$ provided that all the men in the room are hungry. Here the quantifier is taken as restricted to the men in that room. We do not look at wider situations and (possibly) wider extensions of 'men'. So if we take a point $s$ in a Kripke model as a situation rather than a state of information, then it seems we should evaluate the quantifier statically.

Thomason's first-order logic $\mathbf{C F}$ does interpret universal quantifiers statically rather than dynamically. His semantical model is a hybrid of a Kripke model for propositional intuitionistic logic (as the conditional is intuitionistic) and a classical model for predicate logic (as the universal quantifier is static). ${ }^{6}$ Nevertheless, his semantical framework requires different stages to have the same domain. From the standpoint which treats stages as situations, it is obvious that this restriction is inappropriate. From an intuitionistic viewpoint, it is not suitable either. As is well known, Kripke models for intuitionistic logic also require expanding domains. But the connection of intuitionistic logic with expanding domains is both more complicated and more tenuous than is the case with situation theory. In order to see this, let us consider the following schema which we call the distribution schema:

$$
\forall x(\varphi \vee \psi(x)) \supset(\varphi \vee \forall x \psi(x)) \text {, where } x \text { is not free in } \varphi .
$$

If we add to intuitionistic logic all instances of (DS), we obtain a logic whose models are exactly the Kripke models with constant domain. Thus to motivate expanding domains from an intuitionistic viewpoint is to motivate the rejection of this schema.

The BHK interpretation is of little help. According to that we need to show how a proof of $\forall x(\varphi \vee \psi(x))$ could be extended to a proof of $\varphi \vee \forall x \psi(x)$. Well, to have a proof of $\forall x(\varphi \vee \psi(x))$ is to have a construction $C$ which transforms a proof of $a \in D$ ( $D$ the intended range of the variable $x$ ) into a proof of $\varphi \vee \psi(a)$. If the construction $C$ transforms a proof of $a \in D$ into a proof of $\varphi$, then since $x$ is not free in $\varphi$, we would have a proof of $\varphi$. Otherwise, it transforms a proof of $a \in D$ into a proof of $\psi(a)$ and 
thus from the construction $C$ we derive a proof of $\forall x \psi(x)$. Either way we have a proof of $\varphi \vee \forall x \psi(x)$ (for the BHK interpretation, see Troelstra and van Dalen [27], p. 9). The informal semantics of intuitionistic logic does not, at least not obviously, show what is wrong with (DS). Why, then, is (DS) rejected at all? Very briefly, it happens that certain Brouwerian principles of continuity which are more or less selfevident from an intuitionistic standpoint are formally inconsistent in classical logic. These principles say roughly that an assertion about an infinite sequence $\alpha$ must be decided by a finite initial segment of $\alpha$, and hence will be decided the same way for all sequences $\beta$ that agree on that initial segment. Adding (DS) to intuitionistic logic will restore inconsistency with these same principles. Dummett [11] contains a treatment of the semantics of intuitionistic logic which discusses these issues in detail.

There is a further point. Kripke models are not the only semantic structures for intuitionistic logic. Beth trees may be used instead. In the Beth semantics we have a more complicated rule (see Troelstra [26], p. 106) for evaluating disjunctions:

$$
s \models \varphi \vee \psi \text { iff } \forall t \geq s \exists u \geq t(u \models \varphi \text { or } u \models \psi) .
$$

A disjunction is true provided that however knowledge is extended one or other of the disjuncts will become true. With this it is easy to find a counterexample to (DS) that makes no appeal to expanding domains.

The upshot is that expanding domains seem more an artifact of the Kripke semantics than an essential part of the interpretation of intuitionistic logic. However, they are quite central to situation theory, which to some extent supports our choice not to use intuitionistic logic as a basis for situation theory (for more, see Section 4 below).

If we are to allow expanding domains, there is a technical problem to overcome. Specifically, the semantical completeness proof of $\mathbf{C F}$ depends on an auxiliary lemma, that is, Lemma 2 (25, p. 250) and the proof of the lemma in turn makes use of the conditional introduction rule $\supset$ I. However, it is easy to check that if different stages in the semantical models are allowed to have different domains, then the rule is generally not sound since universally quantified sentences, when interpreted statically instead of dynamically, generally are not persistent (see below, $\S \S 2.2$ and 2.5). So, the condition of a constant domain has to be imposed on his models for the sake of CF's semantic completeness; that is to say, in order to have static universal quantifiers, we are forced to adopt a model with constant domain.

Conversely, from the model theoretic standpoint, the models for $\mathbf{C F}$ are a special case of the intuitionistic models. Accordingly, the dynamic condition for quantifiers collapses into the static one. Since the dynamic condition is not suitable and expanding domains are desired as we said above from the situation theoretic viewpoint, it is natural to ask if we can have a logic for situation theory with both static quantifiers and expanding domains.

Motivated by the above, we propose a first-order logical system $\mathbf{C F}^{\prime}$ with strong constructive negation like Thomason's but that allows for expanding domains. Our semantical analysis is still based on Kripke frames $\langle S, \prec, D\rangle$ but we have it in mind to interpret $S$ as a collection of situations rather than conventional possible worlds. Accordingly, $\prec$ is a pre-order on situations and $D$ is a function assigning a set of individuals to each situation. Situations are limited parts of the world. Thus, generally, 
situations provide us with only incomplete information. The partiality of situations to some extent also justifies the use of situations in our semantical framework since, as we pointed out before, constructive logics with strong negation are partial. In addition, we note that another source of the partiality of the logic is from the use of inexact predicates (see [4], Wagner [31], and related citations there). We treat universally quantified sentences statically instead of dynamically. And since static unbounded universally quantified sentences generally are not persistent, we instead consider bounded ones, say $\forall^{\beta} x \varphi(x)$ where $\beta$ is a bounder. This is reminiscent of Devlin's infon logic. Devlin 10 considers $\forall x \in u \sigma$ where $u$ is a set and $\sigma$ is an infon. Such compound infons are persistent because the set $u$ bounds the quantifier. In our framework the bounder $\beta$ may itself be nonpersistent in the sense that the extension of $\beta$ is liable to change from situation to situation, and consequently $\forall^{\beta} x \varphi(x)$ is, in general, not persistent either. Thus, we further distinguish persistent bounders from nonpersistent ones (see Section 2).

We summarize the various approaches to the universal quantifier in the following table, where INT is intuitionistic predicate logic, $\overline{\mathbf{H}}$ is Gurevich's intuitionistic logic with strong negation [17], and $\mathbf{C F}$ is Thomason's first-order logic [25]. For a unifying exposition of both Kripke and Beth models, see van Dalen [28].

\begin{tabular}{|c|c|c|c|}
\hline Logics & Quantifiers $\forall x$ & Models & Domains \\
\hline \hline INT, $\overline{\mathbf{H}}$ & dynamic & Kripke models & expanding \\
INT & static & Beth models & constant \\
CF & static & Kripke models & constant \\
CF $^{\prime}$ & static & Kripke models & expanding \\
\hline
\end{tabular}

In the following, we shall first introduce the logical system $\mathbf{C F}^{\prime}$, then prove its soundness and completeness. Finally, we discuss its connection with situation theory, its possible extensions as well as its potential applications.

\section{Logical system $C F^{\prime}$ with strong constructive negation}

2.1 Language $\mathcal{L}$ of $\boldsymbol{C F}^{\prime} \quad$ The language ${ }^{7}$ of our logical system $\mathbf{C F}^{\prime}$ consists of an infinite set $V_{\mathcal{L}}$ of individual variables (as metavariables for variables we use $x, x_{0}, x_{1}, \ldots$ ), a set $C_{\mathcal{L}}$ of individual constants (metavariables: $c, c_{0}, c_{1}, \ldots$ ), and for each $n, n \geq 0$, a set $P_{\mathcal{L}}^{n}$ of $n$-ary predicate symbols (metavariables: $R_{1}, R_{2}, R_{3}, \ldots$ ). In addition, $\mathcal{L}$ has a set $B_{\mathcal{L}}$ of bounders with a subset $B_{\mathcal{L}}^{P}$ of persistent bounders (metavariables: $\beta, \beta_{0}, \beta_{1}, \ldots$ with or without superscript $P$ ), and a relation symbol $\in$. The set $T_{\mathcal{L}}$ of terms of $\mathcal{L}$ is $V_{\mathcal{L}} \cup C_{\mathcal{L}}$. We use $t, t_{0}, t_{1}, \ldots$ as metavariables for terms.

Atomic formulas of $\mathcal{L}$ are $R\left(t_{1}, t_{2}, \ldots, t_{n}\right)$ and $c \in \beta$, where $t_{1}, t_{2}, \ldots, t_{n} \in$ $T_{\mathcal{L}}, c \in C_{\mathcal{L}}, R \in P_{\mathcal{L}}^{n}$, and $\beta \in B_{\mathcal{L}}$. The well-formed formulas of $\mathcal{L}$ are defined recursively from atomic formulas using the connectives $\vee, \supset$, and $\sim$, and for each bounder $\beta$, a bounded universal quantifier $\forall^{\beta}$ as follows:

1. atomic formulas are formulas;

2. if $\varphi$ is a formula, then so is $\sim \varphi$; 
3. if $\varphi, \psi$ are formulas, then so are $\varphi \vee \psi, \varphi \supset \psi$;

4. if $\varphi$ is a formula, $x$ is a variable, and $\beta$ is a bounder, then $\forall^{\beta} x \varphi(x)$ is also a formula. For simplicity, we write $\forall x \in \beta \varphi(x)$ for $\forall^{\beta} x \varphi(x)$.

A formula of form $\forall x \in \beta \varphi(x)$ is called a bounded, universally quantified formula. Such formulas can be used to express local generality since the bound variables thereof are to range over a subset of the individuals in the universe. In contrast, the generality expressed by unbounded, universally quantified formulas is a kind of overall generality (see Frege [14]). In order to express overall generality by a variable, we only need a device for the scope of the variable, whereas in order to express local generality, we need, in addition, the range of the variable. So, generally speaking, in order to express generality via a variable, we need both a mechanism for the scope of the variable and a parameter for its range. In other words, a logical quantifier consists of the scope of a variable and the range of the variable. From the pragmatic point of view, it is clear that bounded formulas are more frequently used than unbounded ones. In translating natural language, restricted quantifiers are usually represented as unrestricted quantifiers over a material conditional or something equivalent. Thus, 'All birds fly' is formalized as $\forall x(\sim \operatorname{Bird}(x) \vee \operatorname{Flies}(x))$ or $\forall x(\operatorname{Bird}(x) \rightarrow \operatorname{Flies}(x))$, if the material conditional $\rightarrow$ is defined. In $\mathbf{C} \mathbf{F}^{\prime}$ it is represented as $\forall x \in \beta \operatorname{Flies}(x)$, where $\beta$ is a bounder for birds. We prefer our approach to the usual one. In our opinion, it is tidy and emphasizes the two aspects of local generality. More importantly, as we mentioned in the introduction, bounded, universally quantified formulas can be used to express the persistence of information (see below). That is the primary motive for our use of bounded formulas instead of unbounded ones.

Syntactically bounders are flags on quantifiers. Semantically they are to be interpreted as sets, that is, in the same way as predicates are in classical first-order logic. Then, it may be asked, why do we have a special syntax for bounders instead of treating them simply as unary predicates? The answer is that a predicate such as 'Flies $(x)$ ' gives three possibilities: an object may fly, it may not fly, or it may be undecided whether it flies or not. But a bounder supplies only two possibilities: an object is included in the bounder or it is not. The consequence is that $\forall x(\sim \psi(x) \vee \varphi(x))$, in fact, says a little more than $\forall x \in \beta \varphi(x)$ (see $\$ 2.3$ below for exact comparison). It is the latter that captures the informal reading of 'All birds fly' rather than the former.

Conjunction and bounded existential quantification are defined as follows.

$$
\begin{aligned}
& \varphi \wedge \psi==_{\mathrm{df}} \sim(\sim \varphi \vee \sim \psi) . \\
& \exists x \in \beta \varphi(x) \quad=_{\mathrm{df}} \quad \sim \forall x \in \beta \sim \varphi(x) \text {. }
\end{aligned}
$$

The concept of free and bound variables is defined as usual. Bound variables are used as position markers only, and thus $\forall x \in \beta \varphi(x)$ and $\forall y \in \beta \varphi(y)$ would be counted as the same formula. We use as above $\varphi, \psi, \chi, \ldots$ as metavariables for formulas, and $\Gamma, \Delta$ (with or without subscripts) for arbitrary sets of formulas.

2.2 Persistent formulas The concept of persistence comes from situation theory. Informally, it says that what is true in one situation is still true in a larger situation. Formally, there is a so-called persistence principle, stated as

$$
\text { if } s \prec s^{\prime} \text { and } s=\sigma \text {, then } s^{\prime} \models \sigma \text {, }
$$


where $s, s^{\prime}$ are situations, $\sigma$ is an infon, and $\models$ is a support relation between situations and infons. If an infon $\sigma$ satisfies the persistence principle, we say that $\sigma$ is persistent (see Barwise 5]). Generally speaking, universally quantified sentences in natural language are not persistent. 'Everyone here is hungry' may be verified when evaluated from the situation in one poor household, but falsified when evaluated from a larger situation including comfortable ones. There is a tension between quantification and persistence. If we take it that the persistence principle is true of every infon, then it seems universally quantified sentences have to be excluded from the category of infons. And conversely, if universally quantified sentences are taken as infons, then the persistence principle would only hold partially (see [5] pp. 234-36). However, quantified sentences are such important forms for expressing information that they can hardly be excluded from the category of infons. We also want to retain the persistence principle because, as situation theorists have argued, it captures our intuition "that what goes on in part of the world still goes on when one has a broader perspective" (5], p. 236). For the sake of both persistence and a rich algebraic structure of infons, we only consider bounded quantified formulas for which these problems do not arise. However, as we pointed out in the introduction, in our present framework a bounder $\beta$ in $\forall x \in \beta \varphi(x)$ may be nonpersistent. So we introduce an auxiliary notion of persistent bounders. Syntactically, persistent bounders are treated as a primitive notion. Semantic meaning of persistent bounders will be given below (see condition 3 on an interpretation in $\$ 2.3$. Pragmatically, persistent bounders can be obtained by incorporating context into bounders in universally quantified sentences. Then we can define persistent formulas of $\mathcal{L}$ recursively as follows:

1. $R\left(t_{1}, t_{2}, \ldots, t_{n}\right)$ and $\sim R\left(t_{1}, t_{2}, \ldots, t_{n}\right)$ are persistent for any $n$-ary predicate $R$, terms $t_{1}, t_{2}, \ldots, t_{n}$, and $c \in \beta$ and $\sim c \in \beta^{P}$ are persistent for any bounders $\beta$ and $\beta^{P}$;

2. if $\varphi, \psi$ are persistent, then so are $\varphi \vee \psi$ and $\varphi \wedge \psi$;

3. $\varphi \supset \psi$ is persistent for any formulas $\varphi, \psi$;

4. if $\varphi$ is persistent, then $\forall x \in \beta^{P} \varphi(x)$ is persistent;

5. if $\varphi$ is persistent, then $\exists x \in \beta \varphi(x)$ is persistent for any $\beta \in B_{\mathcal{L}}$.

Given a set $\Gamma$ of formulas, let $\Gamma^{P}$ be $\{\varphi \in \Gamma: \varphi$ is persistent $\}$. So, all the persistent formulas of $\mathcal{L}$ would be $F_{\mathcal{L}}^{P}$, where $F_{\mathcal{L}}$ is the set of all $\mathcal{L}$-formulas. Note that, in the definition of persistent formulas, negation is restricted only to atomic formulas. Nevertheless, this will not lose any generality since the negation of a compound formula, according to related rules (see $\$ 2.5$ below), is equivalent to another compound formula in which negation is applied only to atomic formulas.

By the definition, nonpersistence of formulas is only due to the nonpersistence of bounders in universally quantified formulas. So, pragmatically, the persistence of such formulas can be recovered by incorporating context into related bounders. Nevertheless, there exists indeed a kind of unrecoverable nonpersistence. In fact, such nonpersistence is the consequence of the partiality of situations. If a situation is silent on $\sigma$ then it certainly does not preclude a larger more extensive situation settling $\sigma$. In order to express the unrecoverable nonpersistence, we need to add a kind of modal operator such as 'definitely' into our language. Such an extension, however, is outside the scope of this paper (for more, see Mott [20]). The syntactic definition of persis- 
tence will be used in $\$ 2.5$ below.

2.3 Formal semantics Our semantical analysis is essentially similar to Thomason's, but it is based on general Kripke frames instead of particular ones, that is, we allow different points in a Kripke frame to have different domains. A Kripke frame $\mathcal{F}$ is a triple $\langle S, \prec, D\rangle$ such that

1. $S$ is a nonempty set;

2. $\prec$ is a pre-order on $S$, that is, $\prec$ is a reflexive and transitive binary relation on $S$;

3. $D$ is a monotone function assigning sets of individuals to the elements of $S$, that is, for any $s, s^{\prime} \in S$, if $s \prec s^{\prime}$ then $D(s) \subseteq D\left(s^{\prime}\right)$.

$S$ is to be thought of as a set of situations, $\prec$ is the containment relation among situations, and for each $s \in S, D(s)$ is the set of individuals existing at situation $s$.

An interpretation $I$ of language $\mathcal{L}$ on a Kripke frame $\mathcal{F}=\langle S, \prec, D\rangle$ is a function such that: for any $s, s^{\prime} \in S, c \in C_{\mathcal{L}}, R \in P_{\mathcal{L}}^{i}, \beta, \beta^{P} \in B_{\mathcal{L}}$,

1. $I_{s}$ is a partial function from $C_{\mathcal{L}}$ into $D(s)$, and $(a)$ if $s \prec s^{\prime}$ and $I_{s}(c)$ is defined, then $I_{s^{\prime}}(c)$ is also defined and $I_{s}(c)=I_{s^{\prime}}(c)$; and $(b)$ for each $d$ in $D(s), I_{s}(\underline{d})$ is defined and $I_{s}(\underline{d})=d .^{8}$

2. $I_{S}(R)$ is a partial function from the Cartesian product $D(s)^{i}$ into $\{\mathrm{T}, \mathrm{F}\}$, and if $s \prec s^{\prime}$, then $I_{s^{\prime}}(R)$ is an extension of $I_{s}(R)$.

3. $I_{s}$ is a total function from $B_{\mathcal{L}}$ into $\mathcal{P}(D(s))$ such that if $s \prec s^{\prime}$, then $I_{s}(\beta) \subseteq$ $I_{s^{\prime}}(\beta)$ and $I_{s}\left(\beta^{P}\right)=I_{s^{\prime}}\left(\beta^{P}\right)$.

Clause 3 in the definition of interpretation gives us the semantic meaning of persistent sets. In other words, it is the semantic requirement for a set of individuals to be persistent. It is worth pointing out the restriction incorporated in (3) is compatible with the situation theoretic viewpoint, though it may look ad hoc. Anyway, situations are treated as first-class citizens in situation theory. So one possible way to ensure the persistence of universally quantified formulas would be to incorporate reference to situations into them (see [5], p. 236). In this paper, however, we instead adopt the device of persistent bounders.

A Kripke model $\mathcal{M}$ is a pair $\langle\mathcal{F}, I\rangle$ consisting of a Kripke frame $\mathcal{F}$ and an interpretation $I$ on $\mathcal{F}$. Before we continue the formulation of formal semantics, some remarks seem in order about the definition of Kripke models. First, note that, in a Kripke model $\mathcal{M}=\langle S, \prec, D, I\rangle, D(s)$ can be empty for any (and all) $s \in S$. The use of bounders means that the usual restriction to nonempty domains is unnecessary. Thus $\mathbf{C} \mathbf{F}^{\prime}$ is inclusive in the sense that it allows the domain of quantification to be empty (see Bencivenga [9], pp. 379-82).

Second, note that the function $I_{S}\left\lceil C_{\mathcal{L}}\right.$ is partial. So $\mathbf{C} \mathbf{F}^{\prime}$ allows for nondenoting constants just as a free logic does (see [9]). In a free logic, an extra unary predicate $E$ or something equivalent is introduced to deal with reference failure. Nevertheless, in $\mathbf{C} \mathbf{F}^{\prime}$, we do not need such a special predicate. Bounders of quantifiers can play the role of the predicate $E$ of free logic. It may be that bounders are preferable to an existence predicate, at least if one wishes to confine existence to a purely semantic role (as we would). Anyway, it will be no surprise that some axioms and inference rules of $\mathbf{C} \mathbf{F}^{\prime}$ will correspond to axioms and inference rules of a free logic. 
Next, note that the function $I_{S}\left\lceil P_{\mathcal{L}}^{n}(n \geq 0)\right.$ is also partial. That is to say, it may be the case that a basic sentence $R\left(c_{1}, c_{2}, \ldots, c_{n}\right)$ is neither true nor false, so $\mathbf{C F}^{\prime}$ allows truth-value gaps. Such gaps may arise from the use of inexact predicates, but we emphasize that there is another source of truth-value gaps-the partiality of situations.

When a predicate has truth-value gaps, we call it a partial predicate, otherwise a total predicate. A total predicate can be interpreted as a set, that is, in the same way as predicates are in classical first-order logic. With partial predicates, however, we have to associate two sets: one is for the positive assertions, the other for the strong negative assertions. So we might as well divide a partial predicate into two parts, a positive part corresponding to the positive assertions and a negative part corresponding to the strong negative assertions. We recall that, syntactically, bounders are flags on quantifiers. Semantically, as can be seen from clause 3 in the definition of interpretation, bounders are interpreted as sets. What sets, then, should we associate with a bounder $\beta$ ? There are two natural candidates. We could say that $\beta$ was assigned all the objects in the current situation. Then $\forall x \in \beta \varphi(x)$ would be supported by $s$ provided that $s$ made true $\varphi(\underline{a})$ for each object $a$ in $D(s)$. In this case, bounder $\beta$ is nothing more than a denotational variant of the existential predicate $E$ of free logic (see Garson [15], pp. 251-52). An alternative would see bounders in a more restricted way as corresponding to the positive parts of particular predicates, so that $\forall x \in \beta \varphi(x)$ would be interpreted as asserting of all the objects that were $\beta$ in the current situation that they were also $\varphi$. In fact, we choose here not to restrict bounders beyond requiring that the objects a bounder $\beta$ is associated with in a situation $s$ are all objects that belong to the situation $s$.

Given a Kripke model $\mathcal{M}=\langle S, \prec, D, I\rangle$, we define a satisfaction relation $\models_{\mathscr{M}}^{+}$ (or simply $\models^{+}$) and a refutation relation $\models_{\mathcal{M}}^{-}$(or simply $\models^{-}$) between situations $s \in S$ and $\mathcal{L}$-sentences $\varphi$ relative to $\mathcal{M}$ as follows, by induction on the complexity of $\varphi$.

1. $s \models^{+} R\left(c_{1}, c_{2}, \ldots, c_{n}\right)$ iff $I_{s}\left(c_{1}\right), I_{s}\left(c_{2}\right), \ldots, I_{s}\left(c_{n}\right)$ are all defined and $I_{s}(R)\left(I_{s}\left(c_{1}\right), I_{s}\left(c_{2}\right), \ldots, I_{s}\left(c_{n}\right)\right)=\mathrm{T}$;

$s \models-R\left(c_{1}, c_{2}, \ldots, c_{n}\right)$ iff $I_{s}\left(c_{1}\right), I_{s}\left(c_{2}\right), \ldots, I_{s}\left(c_{n}\right)$ are all defined and

$I_{s}(R)\left(I_{s}\left(c_{1}\right), I_{s}\left(c_{2}\right), \ldots, I_{s}\left(c_{n}\right)\right)=\mathrm{F}$;

$s \models+c \in$ iff $I_{s}(c)$ is defined and $I_{s}(c) \in I_{s}(\beta)$;

$s \models{ }^{-} c \in \beta$ iff either $I_{s}(c)$ is not defined or

$I_{s}(c)$ is defined and $I_{s}(c) \in D(s)-I_{s}(\beta)$.

2. $s=^{+} \varphi \vee \psi$ iff $s \models^{+} \varphi$ or $s \models^{+} \psi$;

$s \models^{-} \varphi \vee \psi$ iff $s \models^{-} \varphi$ and $s \models^{-} \psi$.

3. $s \models^{+} \sim \varphi$ iff $s \models^{-} \varphi$;

$s=^{-} \sim \varphi$ iff $s \models^{+} \varphi$.

4. $\quad s \models^{+} \varphi \supset \psi$ iff for all $s^{\prime}$ such that $s \prec s^{\prime}$ if $s^{\prime} \models^{+} \varphi$ then $s^{\prime} \models^{+} \psi$;

$s \models^{-} \varphi \supset \psi$ iff $s \models^{+} \varphi$ and $s \models^{-} \psi$.

5. $\quad s \models^{+} \forall x \in \beta \varphi(x)$ iff for all $d \in D(s)$, if $s \models^{+} \underline{d} \in \beta$ then $s \models^{+} \varphi(\underline{d})$; $s \models^{-} \forall x \in \beta \varphi(x)$ iff for some $d \in D(s), s \models^{+} \underline{d} \in \beta$ and $s \models^{-} \varphi(\underline{d})$.

Basic semantic notions such as consequence, satisfiability, and validity can be defined in the usual way in terms of the satisfaction relation $\models^{+}$. For any sentence $\varphi$ and set $\Gamma$ of sentences, we write $\models \varphi$ to indicate that $\varphi$ is valid, $\Gamma \models \varphi$ to indicate that $\varphi$ is a semantic consequence of $\Gamma$, and $\Gamma \models \Delta$ to indicate that there is a subset $\left\{\varphi_{1}, \varphi_{2}, \ldots, \varphi_{n}\right\}$ of $\Delta$ such that $\varphi_{1} \vee \varphi_{2} \vee \cdots \vee \varphi_{n}$ is a semantic consequence of $\Gamma$. 
Lemma 2.1 (Persistence lemma) Let $\mathcal{M}=\langle S, \prec, D, I\rangle$ be a Kripke model, $\varphi$ a persistent formula of $\mathcal{L}$.

(i) If $s \prec s^{\prime}$ and $s \models^{+} \varphi$ then $s^{\prime} \models^{+} \varphi$.

(ii) If $s \prec s^{\prime}$ and $s \models^{+} \Gamma$ then $s^{\prime} \models^{+} \Gamma^{P}$.

Proof: For (i), routine induction on the complexity of $\varphi$. (ii) is a straightforward corollary of (i).

The persistence lemma 2.1(i) gives us the semantic meaning of persistence. It can be viewed as a variant of the persistence principle.

2.4 Axiomatic system for $\mathbf{C F}^{\prime} \quad$ Our axiomatic system $\mathbf{C F}^{\prime}$ is based on the axiomatic systems for constructive logics with strong negation (see [24], [17], and [2]). It takes as axioms the following list of schemas.

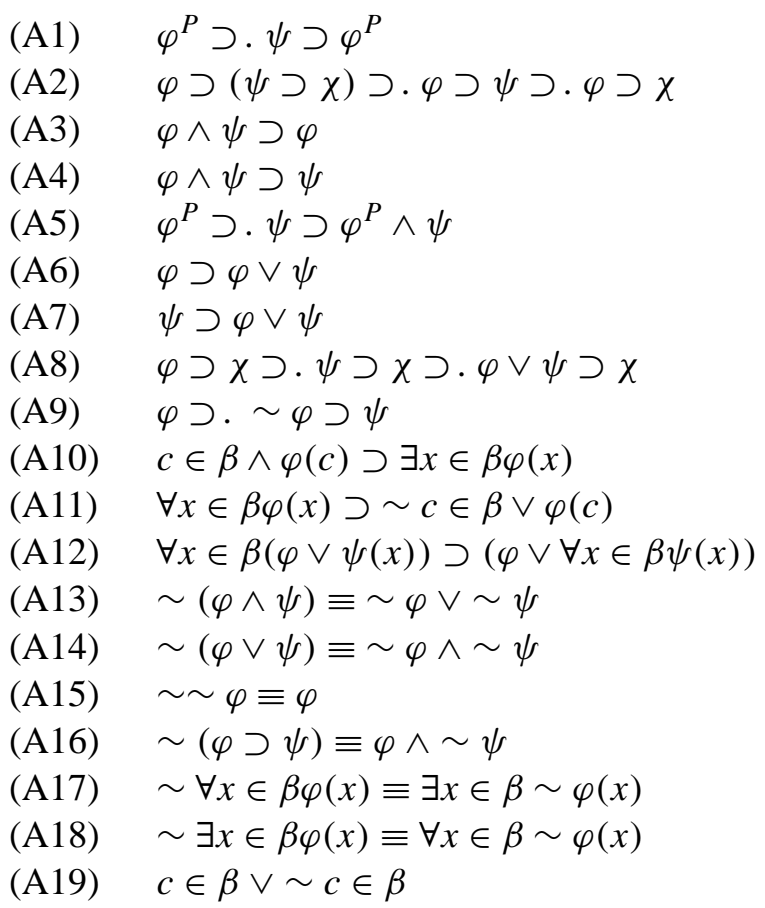

In axioms $\mathrm{A} 1$ and $\mathrm{A} 5, \varphi^{P}$ means that $\varphi$ has to be persistent, which is the little price we have to pay for the relaxation of the dynamic condition on universal quantifiers to the static one. In axiom A12, $x$ is required not to be free in $\varphi$. In addition, note that axiom A12 is not assumed in constructive logics (see [17] and [2]). We emphasize our situation theoretical standpoint rather than intuitionistic or constructive viewpoint. So there seems nothing preventing us from assuming the axiom.

With axiom A19, we are assuming that, at any situation, we can always decide if a constant $c$ is in $\beta$ or not. The assumption is consistent with the semantic interpretation of $\beta$ given above. In addition, note that axioms A13 and A18 can in fact be derived from the other axioms and related definitions and thus can be omitted. 
$\mathbf{C F}^{\prime}$ has the following inference rules.

$$
\begin{gathered}
\frac{\varphi, \varphi \supset \psi}{\psi} \\
\frac{c \in \beta \wedge \varphi(c) \supset \psi}{\exists x \in \beta \varphi(x) \supset \psi} \\
\frac{\psi \supset(\sim c \in \beta \vee \varphi(c))}{\psi \supset \forall x \in \beta \varphi(x)}
\end{gathered}
$$

In rules $\mathrm{R} 2$ and $\mathrm{R} 3$, the constant $c$ is required not to occur in $\psi$.

The axiomatic system $\mathbf{C F}^{\prime}$ is a first-order modification of Almukdad and Nelson's $\mathbf{N}$ as well as Thomason's $\mathbf{C F} .{ }^{9}$ If we delete axiom A9 from $\mathbf{C F}^{\prime}$, denoted $\mathbf{C F}^{\prime-}$, then we have a system which is a modification of Almukdad and Nelson's $\mathbf{N}^{-}$. Since axiom A9 is not available in $\mathbf{C F}^{\prime-}$, we need another axiom to the effect that $c \in \beta$ and $\sim c \in \beta$ do not hold at the same time, say $c \in \beta \wedge \sim c \in \beta \supset \perp$. So, with logic $\mathbf{C F}^{\prime-}$, inconsistent situations are allowed, but the inconsistency of situations does not arise from contradictory statements of the form $c \in \beta \wedge \sim c \in \beta$.

Basic notions (relative to $\mathbf{C F}^{\prime}$ ) such as thesishood, consequence, and consistency can be defined in the usual way. For any sentence $\varphi$ and set $\Gamma$ of sentences, we write $\vdash \varphi$ to indicate that $\varphi$ is a thesis of $\mathbf{C F}^{\prime}, \Gamma \vdash \varphi$ to indicate that $\varphi$ is a consequence in $\mathbf{C F}^{\prime}$ of $\Gamma$, and $\Gamma \vdash \Delta$ to indicate that there is a subset $\left\{\varphi_{1}, \varphi_{2}, \ldots, \varphi_{n}\right\}$ of $\Delta$ such that $\varphi_{1} \vee \varphi_{2} \vee \cdots \vee \varphi_{n}$ is a consequence of $\Gamma$.

From the definitions of thesishood and consequence, it is easy to prove the following lemma.

Lemma 2.2 Let $\Gamma, \Delta$ be sets of $\mathcal{L}$-sentences. If $\Gamma \vdash \Delta$, then $\Gamma^{\prime} \vdash \Delta^{\prime}$ for some finite subsets $\Gamma^{\prime}$ and $\Delta^{\prime}$ of $\Gamma$ and $\Delta$, respectively.

2.5 Derived rules for $\boldsymbol{C F}^{\prime}$ In this section, we list some rules for the deducibilityrelation $\vdash$ of $\mathbf{C} \mathbf{F}^{\prime}$ between sets of $\mathcal{L}$-sentences that are needed in the proof of semantical completeness. It is not difficult to derive them from the axioms and rules of $\mathbf{C F}^{\prime}$ given before. We divide these rules into three groups. Group 1 consists of two structural rules and Group 2 of some operational rules. For $\mathbf{C F}^{\prime-}$, rule $\sim \mathrm{E}$ is to be replaced by a rule equivalent to $c \in \beta \wedge \sim c \in \beta \supset \perp$. Group 3 is about connection between strong negation and other connectives. Lacking the $\sim$-introduction rule, we have to use numerous negation rules to connect negation and other connectives by driving strong negation back and forth across them. Note that, because there is no rule of introduction, we are able to use multiple-conclusion rules without, in general, being able to derive the Law of Excluded Middle (see the related remarks by Gentzen [16], p. 82 and the example about the derivation of the law on p. 85).

\section{Group 1}

R: $\quad$ If $\Gamma$ and $\Delta$ are not disjoint, then $\Gamma \vdash \Delta$.

$$
\mathrm{T}: \quad \frac{\Gamma \vdash \Delta}{\Gamma, \Theta \vdash \Xi, \Delta}
$$




\section{Group 2}

$$
\begin{array}{llll}
\vee \mathrm{I}: & \frac{\Gamma \vdash \varphi, \psi, \Delta}{\Gamma \vdash \varphi \vee \psi, \Delta} & \vee \mathrm{E}: & \frac{\Gamma, \varphi \vdash \Delta ; \Gamma, \psi \vdash \Delta ; \Gamma \vdash \varphi \vee \psi, \Delta}{\Gamma \vdash \Delta} \\
\supset \mathrm{I}: & \frac{\Gamma^{P}, \varphi \vdash \psi}{\Gamma^{P} \vdash \varphi \supset \psi} & \supset \mathrm{E}: & \frac{\Gamma \vdash \varphi \supset \psi, \Delta ; \Gamma \vdash \varphi, \Delta}{\Gamma \vdash \psi, \Delta} \\
& \sim \mathrm{E}: & \frac{\Gamma \vdash \varphi, \Delta ; \Gamma \vdash \sim \varphi, \Delta}{\Gamma \vdash \Delta} \\
\forall \mathrm{I}: \quad \frac{\Gamma \vdash \sim c \in \beta \vee \varphi(c), \Delta}{\Gamma \vdash \forall x \in \beta \varphi(x), \Delta} & \forall \mathrm{E}: & \frac{\Gamma \vdash \forall x \in \beta \varphi(x), \Delta}{\Gamma \vdash \sim c \in \beta \vee \varphi(c), \Delta}
\end{array}
$$

In $\forall \mathrm{I}, c$ has no occurrence in $\varphi(x)$ or in any member of $\Gamma$ or of $\Delta$;

Group 3

$$
\begin{aligned}
& \sim \vee \mathrm{I}: \quad \frac{\Gamma \vdash \sim \varphi, \Delta ; \Gamma \vdash \sim \psi, \Delta}{\Gamma \vdash \sim(\varphi \vee \psi), \Delta} \\
& \sim \vee \mathrm{E}: \quad \frac{\Gamma \vdash \sim(\varphi \vee \psi), \Delta}{\Gamma \vdash \sim \varphi, \Delta} \quad \frac{\Gamma \vdash \sim(\varphi \vee \psi), \Delta}{\Gamma \vdash \sim \psi, \Delta} \\
& \sim \text { I: } \quad \frac{\Gamma \vdash \varphi, \Delta}{\Gamma \vdash \sim \sim, \Delta} \\
& \sim \text { E: } \quad \frac{\Gamma \vdash \sim \sim, \Delta}{\Gamma \vdash \varphi, \Delta} \\
& \sim \mathrm{I}: \quad \frac{\Gamma \vdash \varphi, \Delta ; \Gamma \vdash \sim \psi, \Delta}{\Gamma \vdash \sim(\varphi \supset \psi), \Delta} \\
& \sim \text { E: } \quad \frac{\Gamma \vdash \sim(\varphi \supset \psi), \Delta}{\Gamma \vdash \varphi, \Delta} \quad \frac{\Gamma \vdash \sim(\varphi \supset \psi), \Delta}{\Gamma \vdash \sim \psi, \Delta} \\
& \sim \forall \mathrm{I}: \quad \frac{\Gamma \vdash c \in \beta \wedge \sim \varphi(c), \Delta}{\Gamma \vdash \sim \forall x \in \beta \varphi(x), \Delta} \\
& \sim \forall \mathrm{E}: \quad \frac{\Gamma \vdash \sim \forall x \in \beta \varphi(x), \Delta ; \Gamma, c \in \beta \wedge \sim \varphi(c) \vdash \Delta}{\Gamma \vdash \Delta}
\end{aligned}
$$

Theorem 2.3 (Soundness of $\mathbf{C F}^{\prime}$ ) Let $\Gamma$ be a set of $\mathcal{L}$-sentences, and $\varphi$ an $\mathcal{L}$ sentence, and $\mathcal{M}=\langle S, \prec, D, I\rangle$ a model of $\mathcal{L}$, s a situation in $\mathcal{M}$. If $\Gamma \vdash \varphi$, and $s \models^{+} \Gamma$, then $s \models^{+} \varphi$.

Proof: Proof is routine and thus omitted. 
Note that the soundness of $\mathbf{C} \mathbf{F}^{\prime}$ would fail if we included a rule of $\sim$-introduction $(\sim-\mathrm{I})$ to the effect that from $\Gamma, \varphi \vdash \Delta$ we can infer $\Gamma \vdash \sim \varphi, \Delta$. To see this, observe that, by derived rule $\mathrm{R}$ of $\mathbf{C F}^{\prime}, \varphi \vdash \varphi$. By $\sim$-I it then follows that $\vdash \sim \varphi, \varphi$. And so $\vdash \sim \varphi \vee \varphi$ by rule $\vee$-I. But it is not difficult to see that $\sim \varphi \vee \varphi$ is not valid in the current semantic framework. This shows that $\sim-I$ is not sound in $\mathbf{C} \mathbf{F}^{\prime}$.

\section{Completeness proof of $\mathrm{CF}^{\prime}$}

3.1 Definition A set $\Gamma$ of $\mathcal{L}$-sentences is $\mathcal{L}$ - $\omega$-complete if for all $\mathcal{L}$-formulas $\varphi(x)$, we have $\Gamma \vdash \forall x \in \beta \varphi(x)$ if $\Gamma \vdash \sim c \in \beta \vee \varphi(c)$ for all $c \in C_{\mathcal{L}}$. And $\Gamma$ is $\mathcal{L}$-saturated if it meets the following five conditions: for any $\mathcal{L}$-sentences $\varphi, \psi$,

1. $\Gamma$ is consistent;

2. $\Gamma$ is deductively closed, that is, if $\Gamma \vdash \varphi$, then $\varphi \in \Gamma$;

3. if $\Gamma \vdash \varphi \vee \psi$, then $\Gamma \vdash \varphi$ or $\Gamma \vdash \psi$;

4. if $\sim \forall x \in \beta \varphi(x) \in \Gamma$, then for some constant $c \in C_{\mathcal{L}}, c \in \beta \wedge \sim \varphi(c) \in \Gamma$;

5. $\Gamma$ is $\mathcal{L}$ - $\omega$-complete.

Lemma 3.1 (Saturation lemma I) Let $\Gamma$ be a set of $\mathcal{L}$-sentences, and $\varphi$ an $\mathcal{L}$ sentence. Suppose $\Gamma \nvdash \varphi$. Let $C=\left\{c_{0}, c_{1}, c_{2}, \ldots\right\}$ be a countable set of constants foreign to $\mathcal{L}, B$ a set of bounders of $\mathcal{L} \cup C$, and $\mathcal{L}^{\prime}=\mathcal{L} \cup C \cup B$. Then there is an $\mathcal{L}^{\prime}$-saturated set $\Gamma_{\omega}$ such that $\Gamma \subseteq \Gamma_{\omega}$ and $\Gamma_{\omega} \nvdash \varphi$.

Proof: In order to obtain required $\Gamma_{\omega}$, we define two sequences $\left\langle\Gamma_{i}\right\rangle_{i}$ and $\left\langle\Delta_{i}\right\rangle_{i}$ by induction as follows. Let $\left\langle\varphi_{i}\right\rangle_{i}$ enumerate all $\mathcal{L}^{\prime}$-sentences, and $\left\langle\varphi_{i, 1} \vee \varphi_{i, 2}\right\rangle_{i},\langle\forall x \in$ $\left.\beta_{i} \varphi_{i}(x)\right\rangle_{i}$ and $\left\langle\sim \forall x \in \beta_{i} \varphi_{i}(x)\right\rangle_{i}$ enumerate with infinite repetition all disjunctive, bounded universal and bounded existential sentences of $\mathcal{L}^{\prime}$, respectively.

Let $\Gamma_{0}=\Gamma$ and $\Delta_{0}=\{\varphi\}$. Suppose that $\Gamma_{k}$ and $\Delta_{k}$ have been defined. To define $\Gamma_{k+1}$ and $\Delta_{k+1}$, we distinguish the following five cases.

Case 1: $\quad k=4 n, \Gamma_{k} \vdash \varphi_{n, 1} \vee \varphi_{n, 2}$, and $\varphi_{n, 1} \notin \Gamma_{k}$ and $\varphi_{n, 2} \notin \Gamma_{k}$. Put

$$
\begin{aligned}
\Gamma_{k+1} & =\Gamma_{k} \cup\left\{\varphi_{n, i}\right\}, \\
\Delta_{k+1} & =\Delta_{k},
\end{aligned}
$$

where $i$ is the least of $\{1,2\}$ such that $\Gamma_{k} \cup\left\{\varphi_{n, i}\right\} \forall \Delta_{k}$.

Case 2: $\quad k=4 n+1 . \Gamma_{k} \vdash \sim \forall x \in \beta_{n} \varphi_{n}(x), \Delta_{k}$ and for all constants $c \in C_{\mathcal{L}^{\prime}},(c \in$ $\left.\beta_{n} \wedge \sim \varphi_{n}(c)\right) \notin \Gamma_{k}$. Put

$$
\begin{aligned}
\Gamma_{k+1} & =\Gamma_{k} \cup\left\{c_{k} \in \beta_{n} \wedge \sim \varphi_{n}\left(c_{k}\right)\right\}, \\
\Delta_{k+1} & =\Delta_{k},
\end{aligned}
$$

where $c_{k}$ is the first member of $C_{\mathcal{L}^{\prime}}$ not to occur in $\varphi_{n}(x)$ or in any member of $\Gamma_{k}$ or of $\Delta_{k}$.

Case 3: $\quad k=4 n+2$; there are two subcases.

Subcase 1: $\quad \Gamma_{k}, \varphi_{n} \vdash \Delta_{k}$. Put

$$
\begin{aligned}
\Gamma_{k+1} & =\Gamma_{k}, \\
\Delta_{k+1} & =\Delta_{k} \cup\left\{\varphi_{n}\right\} ;
\end{aligned}
$$


Subcase 2: $\varphi_{n} \notin \Gamma_{k}$ and $\Gamma_{k}, \varphi_{n} \nvdash \Delta_{k}$. Put

$$
\begin{aligned}
\Gamma_{k+1} & =\Gamma_{k} \cup\left\{\varphi_{n}\right\}, \\
\Delta_{k+1} & =\Delta_{k} .
\end{aligned}
$$

Case 4: $\quad k=4 n+3 . \Gamma_{k}, \forall x \in \beta_{n} \varphi_{n}(x) \vdash \Delta_{k}$, and for all constants $c \in C_{\mathcal{L}^{\prime}},(\sim c \in$ $\left.\beta_{n} \vee \varphi_{n}(c)\right) \notin \Delta_{k}$. Put

$$
\begin{aligned}
\Gamma_{k+1} & =\Gamma_{k}, \\
\Delta_{k+1} & =\Delta_{k} \cup\left\{\sim c_{k} \in \beta_{n} \vee \varphi_{n}\left(c_{k}\right)\right\},
\end{aligned}
$$

where $c_{k}$ is the first member of $C_{L^{\prime}}$ not to occur in $\varphi_{n}(x)$ or in any member of $\Gamma_{k}$ or of $\Delta_{k}$.

Case 5: None of the cases above applies. Put

$$
\begin{aligned}
\Gamma_{k+1} & =\Gamma_{k}, \\
\Delta_{k+1} & =\Delta_{k} .
\end{aligned}
$$

It is then not difficult to check by induction that for any $k \in \omega, \Gamma_{k} \not \forall \Delta_{k}$ using the derived rules for $\mathbf{C F}^{\prime}$. To illustrate, let us consider Case 3.1. We need to show that if $\Gamma_{k}, \varphi_{n} \vdash \Delta_{k}$, then $\Gamma_{k} \nvdash \Delta_{k} \cup\left\{\varphi_{n}\right\}$. Suppose $\Gamma_{k} \vdash \Delta_{k} \cup\left\{\varphi_{n}\right\}$. We assume that $\varphi_{n}, \Delta_{k}$ and $\Delta_{k} \cup\left\{\varphi_{n}\right\}$ are the same set of formulas. By rule $\mathrm{T}$ and rule $\vee \mathrm{I}$, we have $\Gamma_{k} \vdash$ $\varphi_{n} \vee \varphi_{n}, \Delta_{k}$. Since we are assuming that $\Gamma_{k}, \varphi_{n} \vdash \Delta_{k}$, it follows that $\Gamma_{k} \vdash \Delta_{k}$ by rule $\vee E$. But this contradicts the induction hypothesis. So we have $\Gamma_{k} \forall \Delta_{k} \cup\left\{\varphi_{n}\right\}$.

Now let $\Gamma_{\omega}=\cup\left\{\Gamma_{k}: k \in \omega\right\}$ and $\Delta_{\omega}=\cup\left\{\Delta_{k}: k \in \omega\right\}$. We can show that $\Gamma_{\omega} \not$ $\Delta_{\omega}, \Gamma_{\omega}=F_{\mathcal{L}^{\prime}}-\Delta_{\omega}$ and $\Gamma_{\omega}$ is $\mathcal{L}^{\prime}$-saturated as desired. The details of verification are omitted.

Lemma 3.2 (Saturation lemma II) Let $\Gamma$ be a set of $\mathcal{L}$-sentences, and $\varphi$ and $\psi \mathcal{L}$ sentences, and $B_{\mathcal{L}}^{P}$ all the persistent bounders in $\mathcal{L}$. Suppose $\varphi \supset \psi \notin \Gamma$. Let $C=$ $\left\{c_{0}, c_{1}, c_{2}, \ldots\right\}$ be a countable set of constants foreign to $\mathcal{L}, B$ a set of bounders of $\mathcal{L} \cup C$, and $\mathcal{L}^{\prime}=\mathcal{L} \cup C \cup B$. Then there is an $\mathcal{L}^{\prime}$-saturated set $\Gamma_{\omega}$ such that $\Gamma^{P} \subseteq$ $\Gamma_{\omega}, \varphi \in \Gamma_{\omega}$ but $\psi \notin \Gamma_{\omega}$ and $\left(\sim c_{j} \in \beta_{i}^{P}\right) \in \Gamma_{\omega}$ for any $c_{j} \in C, \beta_{i}^{P} \in B_{\mathcal{L}}^{P}$.

Proof: The proof is similar to that of saturation lemma I except that this time we let $\Gamma_{0}=\Gamma^{P} \cup\{\varphi\} \cup\left\{\sim c_{j} \in \beta_{i}^{P}: c_{j} \in C \& \beta_{i}^{P} \in B_{\mathcal{L}}^{P}\right\}$ and $\Delta_{0}=\{\psi\}$.

3.2 Definition (Canonical model construction) Let $C_{1}, C_{2}, C_{3}, \ldots$ be a countable sequence of disjoint countable sets of constants foreign to $\mathcal{L}$. Let $C_{n}^{*}$ be $C_{1} \cup C_{2} \cup$ $\cdots \cup C_{n}$, and $B_{n}$ a set of bounders of $\mathcal{L} \cup C_{n}^{*}$ such that $B_{l} \subseteq B_{m}$ for any $l \leq m \leq n$. Then for language $\mathcal{L}_{\omega}=\mathcal{L} \cup\left(\cup C_{n}\right) \cup\left(\cup B_{n}\right)$, we can define a Kripke model $\mathcal{M}=$ $\langle S, \prec, D, I\rangle$ as follows.

1. $S$ consists of all $\Gamma$ such that for some $n, \mathcal{L}_{\Gamma}=\mathcal{L} \cup C_{n}^{*} \cup B_{n}$, and $\Gamma$ is $\mathcal{L}_{\Gamma^{-} \text {-saturated. }}$

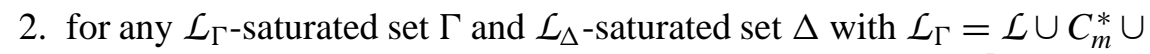
$B_{m}$ and $\mathcal{L}_{\Delta}=\mathcal{L} \cup C_{n}^{*} \cup B_{n}(m<n), \Gamma \prec \Delta$ if and only if $\Gamma^{P} \subseteq \Delta$ and for any $c \in C_{n}^{*}-C_{m}^{*}$ and $\beta^{P} \in B_{\mathcal{L}_{\Gamma}},\left(\sim c \in \beta^{P}\right) \in \Delta$. 
3. if $\Gamma$ is $\mathcal{L}_{\Gamma^{-}}$saturated and $\mathcal{L}_{\Gamma}=\mathcal{L} \cup C_{n}^{*} \cup B_{n}$ then $D(\Gamma)=C_{\mathcal{L}} \cup C_{n}^{*}$.

4. $I_{\Gamma}(c)= \begin{cases}c & \text { if } c \in C_{\mathcal{L}} \cup C_{n}^{*} \\ \text { undefined } & \text { otherwise. }\end{cases}$

5. $I_{\Gamma}(\beta)=\left\{c \in C_{\mathcal{L}} \cup C_{n}^{*}:(c \in \beta) \in \Gamma\right\}$.

6. $I_{\Gamma}(R)\left(c_{1}, c_{2}, \ldots, c_{n}\right)= \begin{cases}\mathrm{T} & \text { if } R\left(c_{1}, c_{2}, \ldots, c_{n}\right) \in \Gamma ; \\ \mathrm{F} & \text { if } \sim R\left(c_{1}, c_{2}, \ldots, c_{n}\right) \in \Gamma ; \\ \text { undefined } & \text { otherwise. }\end{cases}$

Lemma 3.3 (Truth lemma) Suppose $\mathcal{M}=\langle S, \prec, D, I\rangle$ is a canonical Kripke model associated with $\mathcal{L}$. Then for all $\Gamma \in S$, and all $\mathcal{L}_{\Gamma}$-sentences $\chi$, we have

$$
\Gamma \models^{+} \chi \quad \text { iff } \quad \chi \in \Gamma .
$$

Proof: By induction on the complexity of $\chi$.

Case 1: $\chi$ is an atomic sentence $R\left(c_{1}, c_{2}, \ldots, c_{n}\right)$ or $c \in \beta$ : the lemma holds by the definition of a canonical Kripke model.

Case 2: $\chi$ is an atomic sentence $\sim R\left(c_{1}, c_{2}, \ldots, c_{n}\right)$ : the lemma holds again by the definition of a canonical Kripke model. If $\chi$ is $\sim c \in \beta$, suppose that $\Gamma \models^{+} \sim c \in \beta$, that is, $\Gamma \models^{-} c \in \beta$. By definition, either $I_{\Gamma}(c)$ is not defined or $I_{\Gamma}(c)$ is defined and $I_{\Gamma}(c) \notin I_{\Gamma}(\beta)$. In either case, $(c \in \beta) \notin \Gamma$. By axiom A19 and saturatedness of $\Gamma$, we get $(\sim c \in \beta) \in \Gamma$. For converse, let $(\sim c \in \beta) \in \Gamma$. By axiom A9 and the consistency of $\Gamma$, we get $(c \in \beta) \notin \Gamma$. From this it follows that $\Gamma \models^{+} \sim c \in \beta$.

Case 3: $\quad \chi$ is $\varphi \vee \psi$ : the proof is straightforward and thus omitted.

Case 4: $\quad \chi$ is $\sim(\varphi \vee \psi)$ : the proof is straightforward and thus omitted.

Case 5: $\quad \chi$ is $\varphi \supset \psi$. Suppose $\varphi \supset \psi \in \Gamma$. We show $\Gamma \models \varphi \supset \psi$. For any $\Delta$ such that $\Gamma \prec \Delta$, we have $\Gamma^{P} \subseteq \Delta$. Since $\varphi \supset \psi \in \Gamma$ and $\varphi \supset \psi$ is persistent, we get $\varphi \supset \psi \in \Gamma^{P} \subseteq \Delta$. It follows that if $\varphi \in \Delta$, then $\psi \in \Delta$ by rule $\supset \mathrm{E}$. By the hypothesis of induction then, for all such $\Delta$, if $\Delta \models \varphi$, then $\Delta \models \psi$; and therefore $\Gamma \models \varphi \supset \psi$.

Conversely, suppose $\varphi \supset \psi \notin \Gamma$, then $\varphi \supset \psi \notin \Gamma^{P}$, so $\Gamma^{P} \cup\{\varphi\} \forall \psi$ by rule $\supset$ I. Using saturation lemma II, we can get a saturated set $\Delta \in S$ such that $\Gamma \prec \Delta, \varphi \in \Delta$, but $\psi \notin \Delta$. By the hypothesis of induction, we get $\Delta \models \varphi$ but $\Delta \not \psi \psi$. Thus $\Gamma \not \models$ $\varphi \supset \psi$.

Case 6: $\quad \chi$ is $\sim(\varphi \supset \psi) . \Gamma \models^{+} \sim(\varphi \supset \psi)$ if and only if $\Gamma \models^{-} \varphi \supset \psi$ if and only if $\Gamma \models^{+} \varphi$ and $\Gamma \models^{-} \psi$ if and only if $\Gamma \models^{+} \varphi$ and $\Gamma \models^{+} \sim \psi$, and this if and only if $\varphi \in \Gamma$ and $\sim \psi \in \Gamma$ by the hypothesis of induction. But $\varphi \in \Gamma$ and $\sim \psi \in \Gamma$ if and only if $\sim(\varphi \supset \psi) \in \Gamma$ by rules $\sim \supset \mathrm{I}$ and $\sim \supset \mathrm{E}$.

Case 7: $\chi$ is $\sim \sim$ : the proof is straightforward and thus omitted. 
Case 8: $\quad \chi$ is $\forall x \in \beta \varphi(x)$. Suppose $\Gamma \models^{+} \forall x \in \beta \varphi(x)$, then for all $c \in D(\Gamma)$, if $\Gamma \models^{+}$ $c \in \beta$, then $\Gamma \models^{+} \varphi(c)$. But $\Gamma \models^{+} c \in \beta \vee \sim c \in \beta$. It follows that for all $c \in D(\Gamma)$, $\Gamma \models^{+} \sim c \in \beta \vee \varphi(c)$, so $(\sim c \in \beta \vee \varphi(c)) \in \Gamma$ by the hypothesis of induction. Thus $\forall x \in \beta \varphi(x) \in \Gamma$ by the $\mathcal{L}_{\Gamma}-\omega$-completeness of $\Gamma$. Conversely, suppose $\forall x \in \beta \varphi(x) \in$ $\Gamma$, then for any $c \in D(\Gamma),(\sim c \in \beta \vee \varphi(c)) \in \Gamma$ by rule $\forall \mathrm{E}$, so $\sim c \in \beta \in \Gamma$ or $\varphi(c) \in \Gamma$ by the saturatedness of $\Gamma$. Since $\Gamma$ is consistent, if $c \in \beta \in \Gamma$, then $\sim c \in \beta \notin \Gamma$, so $\varphi(c) \in \Gamma$. That is, for any $c \in D(\Gamma)$, if $\Gamma \models^{+} c \in \beta$ then $\Gamma \models^{+} \varphi(c)$ by the hypothesis of induction, so $\Gamma \models^{+} \forall x \in \beta \varphi(x)$.

Case 9: $\chi$ is $\sim \forall x \in \beta \varphi(x)$. The proof is similar to that for Case 8 except that we use condition 4 of $\mathcal{L}_{\Gamma}$-saturatedness of $\Gamma$ and rule $\sim \forall$ I, completing the proof.

Theorem 3.4 (Strong completeness for $\mathbf{C F}^{\prime}$ ) Let $\varphi$ be an $\mathcal{L}$-sentence and $\Gamma$ a set of $\mathcal{L}$-sentences. If $\Gamma \models \varphi$ then $\Gamma \vdash \varphi$.

Proof: $\quad$ Suppose $\Gamma \nvdash \varphi$. By canonical model construction, we can associate $\mathcal{L}_{\Gamma}$ with a canonical Kripke model $\mathcal{M}=\langle\mathcal{S}, \prec, \mathcal{D}, I\rangle$. Saturation lemma I then guarantees us that there is a $\Delta \in S$ such that $\Gamma \subseteq \Delta$ and $\varphi \notin \Delta$. By the truth lemma, $\Delta \models \Gamma$ but $\Delta \not \forall \varphi$. Therefore, $\Gamma \not \models \varphi$.

4 Conclusion and discussion The main contribution of this paper is the proposal of a first-order logic that is based on constructive logic with strong negation. However, different from constructive logic, quantifiers in our system, as in Thomason's, are static rather than dynamic. Our intention is to develop $\mathbf{C F}^{\prime}$ further so that it can serve as a logic for situation theory.

Originally, situation theorists were not much concerned with developing their own logical systems. Their semantic theory of consequence emphasized the external significance of language and the role of nonlinguistic contexts. Consequence is for them no longer a relation between syntactic elements. There is no exact correspondence between the information conveyed by an utterance and the sentence used to convey. In fact " . . . there can be no syntactic counterpart, of the kind traditionally sought in proof theory and theories of logical form, to the [situation] semantic theory of consequence." (see Barwise and Perry [8, pp. 44-45). However, the desire to use situation theory and situation semantics to give an account of inference eventually led Barwise and Etchemendy to construct a situation theoretical model of inference, emphasizing information content. They called this infon logic; that is, a logic whose elementary formulas represent items of information and whose compounds correspond to ways of compounding those items (see Barwise and Etchemendy [7], [10]).

An infon algebra $\mathbb{I}=\langle$ Sit $, I, \Rightarrow, \models\rangle$ consists of a nonempty collection Sit of situations, a distributive lattice $\langle I, \Rightarrow\rangle$ on infons, together with the makes-factual or support relation $\models$ between situations and infons satisfying certain additional conditions.

In an infon algebra $\mathbb{I}$, infons represent pieces of information and situations are intended to be limited portions of the world. The support relation $\models$ is essentially partial: a situation may support some infons and refute others but remains silent on 
many. It follows that any algebraic theory of infons is definitely not Boolean. Furthermore, they argue that a situation theoretical model of infons is at least a complete distributive lattice, that is, a Heyting algebra. Thus, the logic for situation theory is at least intuitionistic but not classical.

This argument immediately poses at least two questions. One of them is about negation, the other about the interpretation of quantifiers. Let us first consider the question about negation.

We recall that in situation theory there are two kinds of basic infons: one is $\left\langle\left\langle R, a_{1}, a_{2}, \ldots, a_{n} ; 1\right\rangle\right\rangle$, the other $\left\langle\left\langle R, a_{1}, a_{2}, \ldots, a_{n} ; 0\right\rangle\right\rangle$, where $R$ is an $n$-place relation, $a_{1}, a_{2}, \ldots, a_{n}$ are objects with the restriction of appropriateness. Note that $a_{1}, a_{2}, \ldots, a_{n}$ need not necessarily be individuals. 0 and 1 are the polarity of infons. For basic infons, negation is defined through a dual operation as follows:

$$
\begin{aligned}
& \overline{\left\langle\left\langle R, a_{1}, a_{2}, \ldots, a_{n} ; 1\right\rangle\right\rangle}=\left\langle\left\langle R, a_{1}, a_{2}, \ldots, a_{n} ; 0\right\rangle\right\rangle ; \\
& \overline{\left\langle\left\langle R, a_{1}, a_{2}, \ldots, a_{n} ; 0\right\rangle\right\rangle}=\left\langle\left\langle R, a_{1}, a_{2}, \ldots, a_{n} ; 1\right\rangle\right\rangle .
\end{aligned}
$$

So, we have

$$
\begin{aligned}
& \overline{\overline{\left\langle\left\langle R, a_{1}, a_{2}, \ldots, a_{n} ; 1\right\rangle\right\rangle}}=\left\langle\left\langle R, a_{1}, a_{2}, \ldots, a_{n} ; 1\right\rangle\right\rangle ; \\
& \overline{\overline{\overline{\left\langle\left\langle R, a_{1}, a_{2}, \ldots, a_{n} ; 1\right\rangle\right\rangle}}}=\overline{\left\langle\left\langle R, a_{1}, a_{2}, \ldots, a_{n} ; 1\right\rangle\right\rangle} .
\end{aligned}
$$

However, it is well known that intuitionistic negation does not satisfy (3) though it satisfies (4).

Furthermore, the negation of compound infons in situation theory is defined by the following version of DeMorgan's laws (see [5], p. 235 and Fernando [12], p. 108). Even in [7] (p. 55), Barwise and Etchemendy do mention that (5) is sometimes assumed in situation theory. However, (5) does not hold though (6) does for intuitionistic negation.

$$
\begin{aligned}
& \overline{\sigma \wedge \tau}=\bar{\sigma} \vee \bar{\tau} \\
& \overline{\sigma \vee \tau}=\bar{\sigma} \wedge \bar{\tau}
\end{aligned}
$$

Therefore, we conclude that situation theoretic negation is not intuitionistic. Moreover, the above way of treating negation by situation theorists to some extent suggests that the negation used in situation theory is in fact strong negation. More importantly, we can put aside the question whether situation theoretic negation is intuitionistic or strong since it turns out that intuitionistic negation can in fact be simulated by strong negation (see [19] and [2]).

Now we consider the question of quantifiers. Quantification of infons is not treated in Barwise and Etchemendy's infon algebra. Presumably, they would not interpret quantifiers dynamically for the reasons we discussed previously. Moreover, quantifiers in related situation theoretical literature are interpreted in one way or another statically rather than dynamically (see [5], p. 271; 10], pp. 134-36; and [12], p. 109). 
Therefore, we are inclined to use constructive negation, more generally, to use constructive logic with strong negation as the underlying logic for situation theory but to interpret quantifiers statically instead of dynamically. That is the way we arrive at the logic $\mathbf{C} \mathbf{F}^{\prime}$ from situation theorists' work on infon logic. However, we do not claim that our logic is fully-fledged. For one thing, the components in a basic formula $R\left(a_{1}, a_{2}, \ldots, a_{n}\right)$, or using the notation of infon logic, $\left\langle\left\langle R, a_{1}, a_{2}, \ldots, a_{n} ; i\right\rangle\right\rangle$ are still individuals whereas infon logic allows them to be any objects. Nevertheless, we do intend to claim that our logic preserves many features of infon logic since (1) $\mathbf{C F}^{\prime}$ is partial in the sense that a formula can be neither true nor false; (2) it has a rich algebraic structure of persistent formulas; (3) with strong negation available, $\mathbf{C F}^{\prime}$ has in fact two kinds of basic formulas very similar to the two kinds of basic infons of situation theory; (4) the negation of compound formulas satisfies DeMorgan's laws which are assumed to hold in situation theory; and (5) quantifiers in $\mathbf{C} \mathbf{F}^{\prime}$ are static, as is consistent with situation theoretical interpretation of quantifiers.

$\mathbf{C F}^{\prime}$ can be extended in many ways. A natural extension is to replace basic formulas $R\left(a_{1}, a_{2}, \ldots, a_{n}\right)$ of $\mathbf{C F}^{\prime}$ with basic infons $\left\langle\left\langle R, a_{1}, a_{2}, \ldots, a_{n} ; i\right\rangle\right\rangle$, emphasizing that components $a_{1}, a_{2}, \ldots, a_{n}$ in basic infons can be any objects not just individuals. Such structures lend themselves to the treatment of complex objects.

Another possible extension is to incorporate an operator into $\mathbf{C} \mathbf{F}^{\prime}$ in order to express nonpersistence. ${ }^{10}$ What is true in one situation is still true in a larger one. However, what is undetermined in a situation may become true or false when more information is available. It is then natural to introduce an operator such as 'definitely' (see [20]) or, more directly, an 'undetermined' operator $U$. Using this operator $U$, the indeterminacy of both the assertion and the (strong) negation of an infon $\sigma$ can be expressed by means of $U \sigma$ and $U \sim \sigma$, respectively. If an agent, querying a situation $s$ for a decision whether $\sigma$, fails to establish both $\sigma$ and $\sim \sigma$, s/he can then thereby establish $U \sigma$. In a larger situation, however, what is originally absent in a smaller situation may become available, thus the same agent may verify $\sigma$ so that $U \sigma$ is rejected. So, $U \sigma$ is not persistent. Similarly, if a query to a situation $s$ fails to refute $\sigma$, then it rejects the claim that $\sigma$ is refuted by $s$ and thereby establishes $U \sim \sigma$. For the same reason, $U \sim \sigma$ is not persistent either. The distinction between strong negation and $U$ is similar to Barwise and Etchemendy's distinction between negation and denial (see Barwise and Etchemendy [6]). However, our approach is radically different from Barwise and Etchemendy's. Among other things, the inclusion of $U$ in our logic will lead us into nonmonotonic logic whereas Barwise and Etchemendy claim that "Closing the class of propositions under conjunction, disjunction, and denial would result in a notion of proposition whose logic is entirely classical" (6, p. 169). Full details of such an extension remain to be done.

In addition to the foundational role for situation theory, $\mathbf{C F}^{\prime}$ may have potential applications in database theory. In database theory, we are concerned with what information we can get from a query to a database. Since data in relational databases are all positive, we have to use the closed world assumption (CWA) (see Reiter [23]) to obtain negative information. For complete databases, CWA is efficient. However, databases often provide us with just an incomplete description of the world. As a result, the use of CWA may give rise to unpleasant consequences (see Abiteboul, Hull, and Vianu [1], p. 282). Thus, much effort has been devoted to ways of deal- 
ing with various kinds of negative information. Among other things, it is argued that strong negation is necessary in many important applications (see [31] and Alferes and Pereira 3]). ${ }^{11}$ Moreover, there is a more general problem to be considered. It is well known that the relational model for database theory is based on first-order logic. However, such a logical foundation is perhaps inappropriate. First of all, the principle of excluded middle is no longer valid when databases are incomplete. Second, though it is always desirable to have the principle of noncontradiction, we certainly do not want the destructive consequences that propositional logic gives. Indeed, from the informational point of view, it is obvious that the inferential rule of form $p \wedge \sim p \rightarrow q$ should always be rejected. As a result, what remains is at most the constraints of form $p \wedge \sim p \rightarrow \perp$. So it is worth seeking a modified foundation for database theory.

Acknowledgments The first author wishes to acknowledge funding from an Overseas Research Students (ORS) Award (\#ORS-95023007), a Tetley \& Lupton Scholarship, a Leeds University Foundation Scholarship, and a departmental scholarship. We should both like to thank anonymous referees for their comments, criticisms, and suggestions which helped greatly to improve the paper.

\section{NOTES}

1. Two similar systems $\mathbf{F}$ and $\mathbf{G}$, or equivalently, $\mathbf{H F}$ and $\mathbf{H G}$ (using Routley's notation), have been studied by Fitch 13. For the difference between Fitch's systems and Nelson's systems, see [24], and see also Thomason's footnote on page 255 of [25].

2. In 4], Almukdad and Nelson use $\mathbf{N}$ and $\mathbf{N}^{-}$for their first-order systems, where $\mathbf{N}^{-}$is the proper subsystem of $\mathbf{N}$ without the axiom schema $\varphi \supset(\sim \varphi \supset \psi)$.

3. Wansing uses $\mathbf{N}^{-}$and $\mathbf{N}$ instead of $\mathbf{N}$ and $\mathbf{N}_{\mathbf{1}}$ respectively. $\mathbf{N}^{-}$and $\mathbf{N}$ are formulated in symmetrical sequent calculus (see [32, pp. 24-25).

4. The terminology of the heredity of information is used in 32] whereas the persistence of information is the situationists' parlance. Note that 32 is only concerned with propositional logics. For the property of predicate logic, see Lemma 3.1 on p. 53 of 17 ]. In intuitionistic logic, the property is called monotonicity (see the lemma on p. 78 of [27].).

5. Hereafter, we use $\underline{a}$ as a name for $a$.

6. It should be pointed out that his model for propositional logic, strictly speaking, is not intuitionistic since the falsity of an atomic sentence at a stage of construction is treated as being discovered directly rather than being decided by later stages.

7. Function symbols introduce nothing new. For simplicity, we avoid them here.

8. We are assuming that every object has a name. In effect we work with the expansion of language $\mathcal{L}$ to accommodate all the objects of all the domains.

9. Note that neither $\mathbf{N}$ nor $\mathbf{C F}$ is formulated in axiomatic formalism.

10. Readers are invited to refer to Veltman's paper "Defaults in update semantics" 291. There he introduces operators such as 'presumably' to deal with nonpersistence within the framework of update semantics. 
11. In [3], exactly speaking, Alferes and Pereira use explicit negation instead of strong negation.

\section{REFERENCES}

[1] Abiteboul, S., R. Hull, and V. Vianu, Foundations of Databases, Addison-Wesley, Reading, 1995.Zbl 0848.68031 4

[2] Akama, S., "Constructive predicate logic with strong negation and model theory," Notre Dame Journal of Formal Logic, vol. 29 (1988), pp. 18-27.Zbl 0647.03005 MR 89k:03026 1,2.4,2.4,4

[3] Alferes, J. J., and L. M. Pereira, Reasoning with Logic Programming, Lecture Notes in Artificial Intelligence 1111, Springer-Verlag, Berlin, 1996. MR 98k:680314.4

[4] Almukdad, A., and D. Nelson, "Constructible falsity and inexact predicates," The Journal of Symbolic Logic, vol. 49 (1984), pp. 231-33.Zbl 0575.03016|MR 86c:03020 1. 1,4

[5] Barwise, J., The Situation in Logic, CSLI Lecture Notes 17, CSLI, Stanford, 1989. Zbl 0664.03003|MR 90g:03033 2.2,2.2,2.2,2.3,4,4

[6] Barwise, J., and J. Etchemendy, The Liar: An Essay on Truth and Circularity, Oxford University Press, Oxford, 1987. Zbl 0678.03001|MR 88k:03009 4,4

[7] Barwise, J., and J. Etchemendy, "Information, infons, and inference," pp. 33-78 in Situation Theory and Its Applications, vol. 1, edited by R. Cooper, K. Mukai, and J. Perry, CSLI Lecture Notes 22, CSLI, Stanford, 1990. 4. 4

[8] Barwise, J., and J. Perry, Situations and Attitudes, The MIT Press, Cambridge, 1983. Zbl 0946.03007|MR 2001h:03051 4

[9] Bencivenga, E., "Free logics," pp. 373-426 in Alternatives in Classical Logic, vol. 3, Handbook of Philosophical Logic, edited by D. Gabbay and F. Guenthner, D. Reidel, Dordrecht, 1985.Zbl 0875.03020 2.3.2.3

[10] Devlin, K., Logic and Information, Cambridge University Press, Cambridge, 1991. Zbl 0732.03001|MR 93c:68094,1,4,4,

[11] Dummett, M., Elements of Intuitionism, Oxford Logic Guides, Clarendon Press, Oxford, 1977.Zbl 0358.02032|MR 58:16194 1

[12] Fernando, T., "On the logic of situation theory," pp. 97-116 in Situation Theory and Its Applications, vol. 1, edited by R. Cooper, K. Mukai, and J. Perry, CSLI Lecture Notes 22, CSLI, Stanford, 1990. MR 1260728 4.4

[13] Fitch, F. B., Symbolic Logic, Ronald Press, New York, 1952. Zbl 0049.00504 MR 15.592n 4

[14] Frege, G., "Begriffsschrift (Chapter I)," pp. 1-20 in Translations from the Philosophical Writings of Gottlob Frege, edited by P. Geach and M. Black, Basil Blackwell, Oxford, 1952.

[15] Garson, J. W., "Quantification in modal logic," pp. 249-307 in Extensions of Classical Logic, vol. 2, Handbook of Philosophical Logic, edited by D. Gabbay and F. Guenthner, D. Reidel, Dordrecht, 1985. Zbl 0875.03050||MR 844600 2.3 
[16] Gentzen, G., "Investigations into logical deduction," pp. 68-131 in The Collected Papers of Gerhard Gentzen, edited by M. E. Szabo, North-Holland, Amsterdam, 1969. 2.5

[17] Gurevich, Y., "Intuitionistic logic with strong negation," Studia Logica, vol. 36 (1977), pp. 49-59. Zb1 0366.02015 MR 58:160 1.1.1.12.4.2.4.4.

[18] Lopez-Escobar, E. G. K., "Refutability and elementary number theory," Indagationes Mathematicae, vol. 34 (1972), pp. 362-74.ZZbl 0262.02027MR 48:10772 1

[19] Markov, A. A., "Constructive logic" (in Russian), Uspekhi Matematičeskih Nauk, vol. 5 (1950), pp. 187-88. 1,4

[20] Mott, P. L., “Intuitionistic logic with a 'definitely' operator,” Research Report 97.05, School of Computer Studies, University of Leeds, 1997. 2.2.1.

[21] Nelson, D., "Constructible falsity," The Journal of Symbolic Logic, vol. 14 (1949), pp. 16-26. Zbl 0033.24304 MR 10.669a 1. I

[22] Rasiowa, H., " $\mathcal{N}$-lattices and constructive logic with strong negation," Fundamenta Mathematicae, vol. 46 (1958), pp. 61-80.Zbl 0087.00905 MR 20:5137 1

[23] Reiter, R., "On closed world databases," pp. 55-76 in Logic and Databases, edited by H. Gallaire and J. Minker, Plenum Press, New York, 1978. 4

[24] Routley, R., "Semantical analyses of propositional systems of Fitch and Nelson," Studia Logica, vol. 33 (1974), pp. 283-98. Zbl 0356.02022 MR 51:70 1.12.4.4.

[25] Thomason, R. H., "A semantical analysis of constructible falsity," Zeitschrift für mathematische Logik und Grundlagen der Mathematik, vol. 15 (1969), pp. 247-57. 1.,1, $1,11,4$

[26] Troelstra, A. S., Choice Sequences: A Chapter of Intutitionistic Mathematics, Oxford Logic Guides, Clarendon Press, Oxford, 1977.ZZbl 0355.02026 MR 57:15979 1

[27] Troelstra, A. S., and D. van Dalen, Constructivism in Mathematics: An Introduction, vol. 1, North-Holland, Amsterdam, 1988. Zbl 0653.03040 MR 90e:03002a 1,4

[28] van Dalen, D. "Intuitionistic logic", pp. 225-339 in Alternatives in Classical Logic, vol. 3, Handbook of Philosophical Logic, edited by D. Gabbay and F. Guenthner, D. Reidel, Dordrecht, 1985.Zbl 0875.03038 1

[29] Veltman, F., "Defaults in update semantics," Journal of Philosophical Logic, vol. 25 (1996), pp. 221-61.Zbl 0860.03025|MR 97d:68220 4

[30] Vorob'ev, N. N., "Constructive propositional calculus with strong negation" (in Russian), Doklady Akademii Nauk SSSR, vol. 85 (1952), pp. 465-68.

[31] Wagner, G., "Logic programming with strong negation and inexact predicates," Journal of Logic and Computation, vol. 1 (1991), pp. 835-59. Zbl 0738.68018|MR 1165249 1. 4

[32] Wansing, H., Logic of Information Structures, Lecture Notes in Artificial Intelligence 681, Springer-Verlag, Berlin, 1993. Zbl 0788.03001||MR 95b:03035 1, 1, [1, 1, 4, 4, 4,

School of Computer Studies

University of Leeds

Leeds LS2 9JT

UNITED KINGDOM

email: wang@scs.leeds.ac.uk 
School of Computer Studies

University of Leeds

Leeds LS2 9JT

UNITED KINGDOM

email: pmott@scs.leeds.ac.uk 